\title{
Afferents and Homotypic Neighbors Regulate Horizontal Cell Morphology, Connectivity, and Retinal Coverage
}

\author{
Benjamin E. Reese, Mary A. Raven, and Stephanie B. Stagg \\ Neuroscience Research Institute and Department of Psychology, University of California at Santa Barbara, Santa Barbara, California $93106-5060$
}

\begin{abstract}
Horizontal cells are inhibitory interneurons with laterally oriented dendrites that overlap one another, contacting the pedicles of cone photoreceptors. Because of their regular spacing, the network of horizontal cells provides a uniform coverage of the retinal surface. The developmental processes establishing these network properties are undefined, but cell-intrinsic instructions and interactions with other cells have each been suggested to play a role. Here, we show that the intercellular spacing of horizontal cells is essentially independent of genetic background and is predicted by local density, suggesting that horizontal cell positioning is modulated by proximity to other horizontal cells. Dendritic field area compensates for this variation in intercellular spacing, maintaining constant dendritic coverage between strains. Functional dendritic overlap is achieved anatomically at the level of the pedicles, where horizontal cells interact with one another to establish their connectivity: the number of dendritic terminals contacting a pedicle changes, reciprocally, between neighboring horizontal cells during development based on their relative proximity to each pedicle. Cellular morphology is also shown to be regulated by the afferents themselves: afferent elimination before innervation does not alter dendritic field size nor stratification but compromises dendritic branching and prevents terminal formation. Afferent and homotypic interactions therefore generate the morphology, spacing, and connectivity of horizontal cells underlying their functional coverage of the retina.
\end{abstract}

Key words: cone photoreceptor; pedicle; Voronoi domain; regularity index; effective radius; dendritic morphology

\section{Introduction}

Horizontal cells give rise to stratifying processes distributed within the outer plexiform layer of the retina. The dendrites of the horizontal cells ramify in a radiate pattern within this synaptic layer, where their sole source of afferent innervation is from cone photoreceptors. Aggregates of fine processes from the horizontal cell dendrites invaginate into each terminal of a cone photoreceptor, the pedicle, and because of their overlapping dendritic spread, multiple horizontal cells contact individual pedicles (for review, see Peichl et al., 1998). How these horizontal cells achieve their adult form, coverage, and connectivity is unknown, but both intrinsic as well as environmental determinants are likely to participate in the establishment of this morphology. Cellintrinsic factors, dependent on a downstream cascade triggered by fate determination events, equip newborn neuroblasts with a complement of transcriptional regulators, membrane receptors, signal transduction components, and cytoskeletal elements that constrain, direct, and mediate the cellular response to environmental signals (Scott and Luo, 2001; Jan and Jan, 2003; Komiyama et al., 2003; Yu and Malenka, 2003). Those signals

Received Nov. 30, 2004; revised Jan. 4, 2005; accepted Jan. 12, 2005.

This work was supported by a grant from the National Institutes of Health (EY-11087). We thank Drs. Jeremy Nathans and Yangshu Wang (Johns Hopkins University, Baltimore, MD) for providing the original coneless transgenic mice to seed our colony, the primer sequence for confirming genotype, and the cone opsin antisera, Dr. Jeremy Cook (University College London, London, UK) for comments on the mosaic analysis, and Dr. Bill Rice (University of California at Santa Barbara, Santa Barbara, (A) for advice on statistical analysis.

Correspondence should be addressed to B. E. Reese, Neuroscience Research Institute, University of California, Santa Barbara, CA 93106-5060. E-mail: breese@psych.ucsb.edu.

D01:10.1523/JNEUROSCI.4876-04.2005

Copyright $\odot 2005$ Society for Neuroscience $\quad$ 0270-6474/05/252167-09\$15.00/0 may include secreted growth factors, extracellular matrix molecules, or membrane-bound or activity-dependent signaling provided by other cells in the vicinity, including their afferents and homotypic neighbors (Cline, 2001; Lom et al., 2002; Wong and Ghosh, 2002; Grueber et al., 2003; Hua and Smith, 2004). Just how much of this developmental program is dependent on interactions with neighboring cells remains to be determined.

At one extreme, horizontal cells, after their birth, may activate a cell-intrinsic program that leads them down a differentiation pathway specifying a dendritic field of a particular size and morphology (Lin et al., 2004), where proximity between neighboring cells is defined by the periodic occurrence of fate-determining events at the time of neurogenesis (Cameron and Carney, 2004). Differential retinal expansion may then create the variation in cell density, and, through interstitial dendritic growth, the corresponding variation in dendritic field size to which density is inversely related (Lia et al., 1987; Wässle et al., 1989; Rodieck and Marshak, 1992; Scheibe et al., 1995; Cellerino et al., 2000). Alternatively, horizontal cells may interact with neighboring cells during development to produce the correlation between intercellular spacing and dendritic field size, whereas their cone afferents may stimulate the detailed formation of their dendritic morphology.

We have addressed these questions by comparing strains of mice that differ in horizontal cell density, by examining the spatial and morphological relationships between dendritic differentiation and afferent innervation at different stages of development, and by determining the effects of afferent elimination before horizontal cell differentiation. We found that intercellular spacing is primarily independent of genetic background, being simply a function of local horizontal cell density. By comparing 
the morphology of horizontal cells in two strains, we found that dendritic field area nearly doubled as density declined by $50 \%$, suggesting that homotypic neighbor relationships constrain dendritic growth, ensuring uniform retinal coverage. We examined the relationship between the number of dendritic terminals contacting a pedicle as a function of eccentric position within the dendritic field, finding that horizontal cells colonized pedicles closer to the soma and relinquished contacts with those at greater distances as development proceeds. Finally, we found that afferent innervation was critical for dendritic patterning and terminal formation but played no role in defining dendritic field size nor stratification. Size, branching, termination pattern, and stratification are, therefore, independently controlled by distinct environmental signals to establish the functional coverage of the retinal surface.

\section{Materials and Methods}

Tissue preparation. A/J, B6AF1/J, 129S3/SvIm/J, and C57BL/6J ${ }^{\text {Tyr+/c2J }}$ mice (abbreviated as A, B6A, 129, and B6) were obtained from The Jackson Laboratory (Bar Harbor, ME) (or breeding pairs were obtained and bred in the Central Vivarium at the University of California at Santa Barbara in the case of the C57BL/6J ${ }^{\mathrm{Tyr}+/ \mathrm{c} 2 \mathrm{~J}}$ mice) and were analyzed for their mosaic regularity and intercellular spacing at 3 weeks of age. The A strain of mice is albino, whereas the B6 mice were heterozygous for a recessive mutation in the tyrosinase gene, but, as indicated previously, albino gene dosage has no effect on either horizontal cell numbers nor on their mosaic regularity or intercellular spacing (Raven and Reese, 2002).

Coneless transgenic mice on a C57BL/6 background were obtained from Dr. Jeremy Nathans (Johns Hopkins University, Baltimore, MD) and were subsequently bred at the University of California at Santa Barbara. Heterozygotes were mated with C57BL/6 mice (Charles Rivers Laboratories, Cambridge, MA), generating mixed litters of wild-type and coneless offspring to compare the effects of afferent ablation on the developing morphology of horizontal cells. DNA was harvested from tail samples from all animals, and genotype was determined independently for each animal by PCR using primers for detecting the transgene sequence (5'-CAAGGAAATTATGACGATGATTGG- $3^{\prime}$ and $5^{\prime}$-GGCTTGAGCCATATACTCATACATCGC-3').

Mature or developing mice were given a lethal dose of sodium pentobarbital $(120 \mathrm{mg} / \mathrm{kg}$, i.p.) and were then intracardially perfused with physiological saline followed by $4 \%$ paraformaldehyde $\left(\mathrm{pH} 7.2\right.$ at $20^{\circ} \mathrm{C}$ in $0.1 \mathrm{M}$ sodium phosphate buffer). Whole retinas were either dissected from the eyes or the entire eye was embedded in sucrose gelatin and sectioned through the optic nerve head at $16 \mu \mathrm{m}$. Retinal sections were prepared for immunohistochemistry, whereas whole retinas were prepared for DiI labeling. A subset of those whole retinas was subsequently embedded in agarose and sectioned at $100 \mu \mathrm{m}$ through the optic nerve head.

Immunolabeling. Whole retinas were labeled with antibodies to calbindin [a mouse monoclonal antibody at 1:10,000 from Sigma (St. Louis, $\mathrm{MO}$ ) or a rabbit polyclonal antiserum at 1:1000 from Oncogene Research Products (La Jolla, CA)] or to cone opsin (being a mixture of rabbit polyclonal antisera to $\mathrm{S}$ cone opsin at 1:50,000 and $\mathrm{M}$ cone opsin at 1:3000 from Jeremy Nathans, Johns Hopkins University). Primary antibodies were detected with secondary IgGs conjugated to Cy2 or Texas Red, as described previously (Raven and Reese, 2003). Retinal sections cut at $16 \mu \mathrm{m}$ were stained using antibodies to calbindin, cone opsin, or neurofilaments [a rabbit polyclonal antiserum to the $150 \mathrm{kDa}$ neurofilament subunit at 1:500-5000 from Chemicon (Temecula, CA)]. In sections, primary antibodies were detected with secondary IgGs conjugated to biotin for subsequent detection using streptavidin-HRP, as described previously (Raven and Reese, 2003).

Mosaic analysis. Six to eight retinal fields were sampled from each retina, coming from either the center (near the optic nerve head) or the periphery (near the margin). Because the densities for the central versus peripheral samples within a strain were not significantly different, they were averaged to obtain an overall estimate of horizontal cell density for each animal. Those animal scores were then averaged to generate a mean density for each strain. The individual fields were sampled using a $20 \times$ objective and a digital camera attached to the fluorescence microscope. The $X-Y$ coordinates of each cell were determined in every sampled field (being 31, 31, 36, and 23 sampled fields from A, B6A, 129, and B6 mice, respectively) using MetaMorph software (Universal Imaging, Downingtown, PA), from which horizontal cell density was determined, and from which Voronoi domain and autocorrelation analyses were then conducted using software programs designed for this purpose (Raven and Reese, 2002). Regularity indexes and effective radii were calculated as described previously (Rodieck, 1991; Raven and Reese, 2002). Cone densities were also sampled (as above), in A and B6 mice, at a location midway between the optic nerve head and retinal periphery in each retinal quadrant, from which an average density was calculated for each animal.

DiI labeling and morphometric analysis. Crystals of the lipophilic dye DiI (Molecular Probes, Eugene, OR) were inserted under microscopic control into the fixed and whole-mounted retinas of adult $(>1$ month of age) B6 and A wild-type mice and into the fixed and whole-mounted retinas of developing and adult wild-type and coneless littermates. Retinas were left under a coverslip in fixative for 1,2 , or $5 \mathrm{~d}$ for postnatal day 5 (P5), P10, or adult retinas, respectively, when individual horizontal cells, labeled by diffusion through their axons (Sandmann et al., 1996), were subsequently harvested using a Bio-Rad (Hercules, CA) 500 or Olympus (Melville, NY) Fluoview laser scanning confocal microscope at either 0.5 or $1.0 \mu \mathrm{m}$ intervals. Individual cells were reconstructed through their full three-dimensional extent using MetaMorph. The number of primary dendrites, the size of the dendritic field area, the number of branch points per dendritic field, and the number of terminal clusters per dendritic field were determined from a minimum of 10 cells in each experimental condition at each age. Morphometric analysis was conducted directly from the reconstruction (for determining dendritic field area) or from the individual confocal planes (for the other three features) using MetaMorph. Because the adult retinas were larger than those used for determining horizontal and cone photoreceptor cell densities (being P21), the average dendritic field area of labeled horizontal cells was scaled by the ratio of retinal areas between these two groups to compare dendritic field area to horizontal cell and cone photoreceptor densities.

Statistical comparisons. The Pearson correlation coefficient was calculated for the dataset relating effective radius (ER) to horizontal cell density across the four different strains of mice (see Fig. 1h). Student's $t$ tests were conducted for the comparisons of dendritic morphology between $\mathrm{A}$ and B6 mice (see Fig. 2e-h) for the comparison of cone densities in A versus B6 mice (Fig. 2i) and for the comparison of cone densities in wild-type versus coneless mice at P5. Student's $t$ test was conducted to compare the slopes for each cell of the number of terminals per cluster as a function of distance from the soma (see Fig. 3). Two-way betweengroups (group $\times$ age) ANOVAs were conducted to determine whether there was an effect of afferent elimination on each of the four measured features of horizontal cell morphology (see Fig. 6); if there was, then post hoc Scheffe tests were conducted to determine the ages showing an effect of group, and one-way ANOVAs were then conducted to determine whether there was an effect of age within the control (wild-type) or experimental (coneless) groups, followed by post hoc Scheffe tests.

\section{Results}

Horizontal cell density is correlated with intercellular spacing In the mouse retina, a single type of axon-bearing horizontal cell is present (Peichl and González-Soriano, 1994), being distributed across the retinal surface in an orderly array (Fig. 1a) with relatively little variation in density associated with retinal eccentricity (Strettoi and Pignatelli, 2000; Raven and Reese, 2002). Between different strains, however, the density of horizontal cells varies conspicuously (Williams et al., 1998), with some strains of mice having twice as many horizontal cells as others (Fig. 1a,b). An index of the regularity of the mosaics, based on an analysis of their Voronoi domains (Fig. 1c), shows relatively little variation be- 

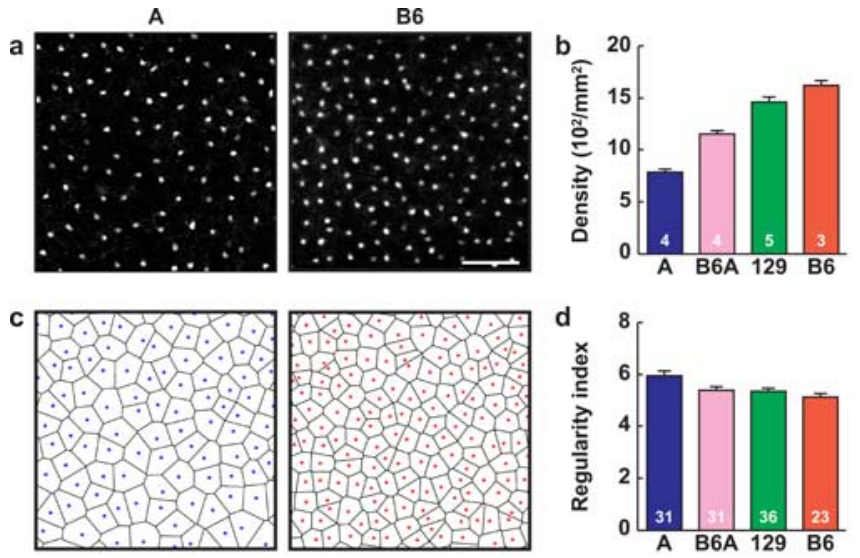

e
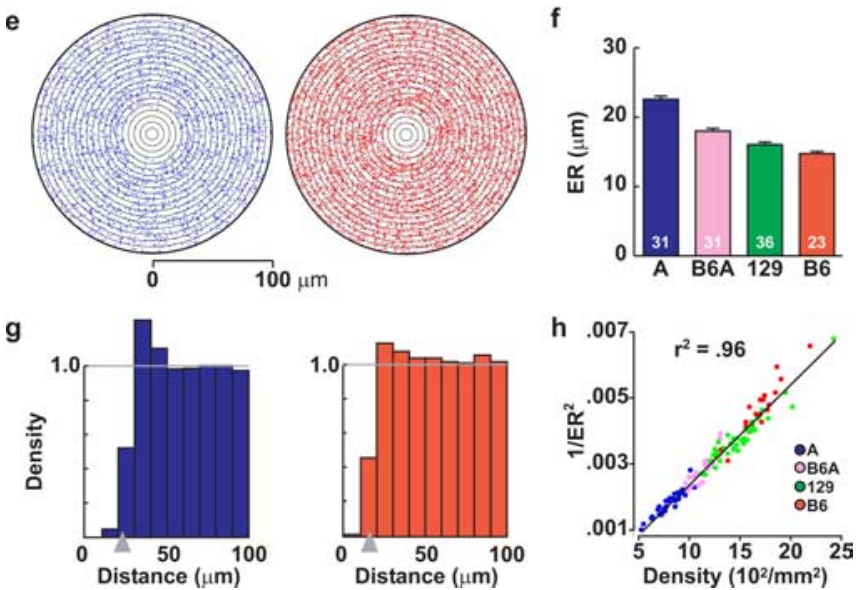

Figure 1. Horizontal cells modulate their intercellular spacing in proportion to local density. $\boldsymbol{a}$, Horizontal cell populations from two strains of mice, A and B6. Scale bar, $100 \mu \mathrm{m}$. $\boldsymbol{b}$, Horizontal cell density shows a twofold variation across four strains of mice. c, Voronoi domain tiling of the retina for these same two samples in $\boldsymbol{a}$. $\boldsymbol{d}$, Regularity index derived from Voronoi domain analysis is relatively invariant across the four strains of mice. $e$, Autocorrelograms for the two fields in $\boldsymbol{a}$, showing a vacant region surrounding the origin which is larger in A relative to $B 6 . f$, ER, calculated from the density recovery profile, shows a conspicuous variation across the four strains of mice. $\boldsymbol{g}$, Normalized density recovery profiles (derived from the autocorrelograms in $\boldsymbol{e}$ ) and their respective effective radii (being $22.8 \mu \mathrm{m}$ for $A$ and $16.1 \mu \mathrm{m}$ for B6; gray arrowheads). $\boldsymbol{h}$, Effective radius varies as a function of density rather than genotype, shown here in the plot of the reciprocal of $E R^{2}$ versus horizontal cell density for each of the individual fields averaged in $\boldsymbol{f}$. $n=$ the number of retinas analyzed in $\boldsymbol{b}$ and the number of fields analyzed from those retinas in $\boldsymbol{d}$ and $\boldsymbol{f}$. Error bars represent SE.

tween strains with indexes generally clustering within the region of 5-6 (Fig. 1d). Rather, autocorrelation analysis (Fig. 1e) reveals that the size of the exclusion zone surrounding individual horizontal cells [the effective radius (Rodieck, 1991)] (Fig. 1g) varies between the strains (Fig. $1 f$ ). When plotted as a function of horizontal cell density, however, the relationship with effective radius is near-perfect across those four strains (Fig. 1h), suggesting that the spacing relationships between horizontal cells are primarily independent of genetic background and are caused by generic intercellular interactions between neighboring horizontal cells that restrict their proximity.

\section{Dendritic field size varies between strains, maintaining constant dendritic coverage}

Given this relationship between horizontal cell spacing and density, dendritic field size should likewise be modulated if these cells are to maintain a constant dendritic coverage. The dendritic fields of individual horizontal cells in the mouse retina approxi- mate circular profiles (Fig. $2 a, b$ ) with, on average, six primary dendrites branching to contact individual cone pedicles within the dendritic field (Fig. $2 c-f$ ). Those fields in mice of the A strain are in fact larger than those in B6 mice, their areas being nearly twice as large (Fig. $2 g$ ). If an average dendritic coverage factor is determined by multiplying average field area by average strain density, values of 6.2 and 6.5 are obtained for these two strains, respectively, indicating that dendritic field area varies to maintain a dendritic overlap of around six or seven cells. These results suggest that dendritic field area, like intercellular spacing, depends on neighbor relationships with other horizontal cells rather than on any genetic differences between the strains intrinsically stipulating size.

Functional dendritic coverage is achieved at the cone pedicles themselves, and the number of terminal clusters (being the sites of pedicle contact) (Fig. $2 c, d$ ) per horizontal cell is identical in A and B6 mice (Fig. 2h). In the presence of equivalent densities of cone photoreceptors, this must mean a declining convergence ratio of horizontal cells onto pedicles. Alternatively, cone density would need to follow horizontal cell density to maintain a constant dendritic coverage at the level of the pedicle. In fact, cone densities in the A strain of mice are 58\% of those in B6 (Fig. 2i), consistent with a conserved functional coverage by the horizontal cells. If the total number of cones falling within the dendritic field of a horizontal cell is calculated, based on the average size of the dendritic field of the horizontal cell and the average cone density, there are 70.7 cones per cell for A and 68.9 cones per cell for B6. Although these numbers are somewhat greater than the number of terminal clusters per cell (Fig. $2 h$ ), they are essentially the same for the two strains, consistent with a conserved functional coverage.

\section{Terminal cluster size changes across the dendritic field during development}

On the basis of this analysis between two strains of mice, A and B6, the numbers of horizontal cells and cone photoreceptors would appear to covary, so that the number of cones contacted by a horizontal cell is about the same in each strain, thereby conserving the number of horizontal cells contacting a cone. Within the dendritic field, mature horizontal cells do not uniformly innervate the cones across the field. Rather, the number of terminals in a cluster innervating a pedicle decreases as a function of pedicle distance from the horizontal cell itself (Ahnelt and Kolb, 1994a). We found that this relationship between terminal number at a pedicle and pedicle distance from the soma is not invariant during development but changes during the period of dendritic differentiation. Whereas mature B6 mouse horizontal cells show a steep falloff in terminal number per pedicle as a function of increasing distance from the soma (Fig. 3a), P10 horizontal cells from B6 mice show a shallow relationship (Fig. $3 b$ ), containing fewer terminals per pedicle, on average, close to the soma and more terminals per pedicle at the extremes of the dendritic field, each relative to the adult. Because of the comparable dendritic overlap between horizontal cells at these two stages (the dendritic coverage factor for P10 horizontal cells being 6.8), the loss of peripheral terminals at a pedicle must therefore be concurrent with the gain of central terminals for another horizontal cell (Fig. 3c).

These results suggest that horizontal cells also interact with their afferents as they differentiate, although it is not clear whether the role played by the afferents is to direct the positioning of the horizontal cell terminals, or if they play a differentiative role, stimulating them to form. Likewise, afferent innervation may be critical for the stratification of the horizontal cell dendrites, directing their coalescence to the future outer plexiform 
layer (OPL). To determine the role of the afferents, we first asked when horizontal cells achieve their characteristic morphology relative to the differentiation of their afferents. We then considered the effects of afferent elimination during early development.

\section{Dendritic stratification proceeds} normally in coneless transgenic mice On the day of birth, horizontal cells are aligned in the outer part of the neuroblast layer, when they have a bipolar morphology, with processes extending primarily in the radial axis (Reese et al., 1999). During the next 4 postnatal days, these cells gradually adopt a stellate morphology, with multiple processes extending out from the soma in most directions, overlapping the dendritic terrains of neighboring horizontal cells (Fig. 4a). Subsequently, the processes of horizontal cells become distributed within the newly differentiating OPL, so that by the second postnatal week, they are restricted to this layer, as they are in maturity (Fig. 4b,c). Cone photoreceptors at birth produce terminals directed toward the row of differentiating horizontal cells. By P5, a continuous row of nascent cone pedicles forms in anticipation of the differentiating OPL (Fig. 4d). These developmental results suggest that the cones may provide an instructive signal for the dendritic stratification of horizontal cells to form.

Transgenic mice expressing an attenuated diphtheria toxin gene under the control of a promoter derived from the human L-cone pigment gene (Soucy et al., 1998) were therefore examined to study the effects of ablating the population of cone photoreceptors on horizontal cell differentiation. Despite the nearcomplete loss of cones in these mice, horizontal cell survival is not affected (Raven and Reese, 2003). Cone density in these transgenic mice is already reduced by $46 \%$ on P5 (mean and SE, $14,400 \pm 504$ cones per $\mathrm{mm}^{2}$ for wild-type retinas vs $7717 \pm 618$ cones per $\mathrm{mm}^{2}$ for coneless littermates; $p<0.01$ ) (Fig. 4e,j), consistent with early opsin expression (Wang et al., 1992; Fei, 2003), and few of those cones still remaining at this age extend terminals that stratify in the region of the nascent OPL (Fig. 4i). Immunopositive cells detach from the outer limiting membrane and translocate into the inner parts of the neuroblast layer (Fig. $4 i$, arrows), a feature not seen in wild-type tissue (Fig. $4 d$ ). These profiles are most frequent at P5, their numbers declining thereafter, and are likely to be dying cones. Despite the loss of these afferents, the coalescence of horizontal cell processes in the OPL is essentially unaffected. At P5, when conspicuous numbers of cones have already been eliminated, the stellate morphology of the horizontal cells is indiscriminable from that of wild-type cells (Fig. 4f). By P9, their stratified organization within the OPL has materialized (Fig. $4 g, h$ ), indicating no concurrent requirement of the afferents for this stratification to form.

\section{Dendritic patterning of horizontal cells requires afferent innervation}

The dendritic stratification of horizontal cells, like the survival of the cells themselves (Raven and Reese, 2003), is apparently inde-
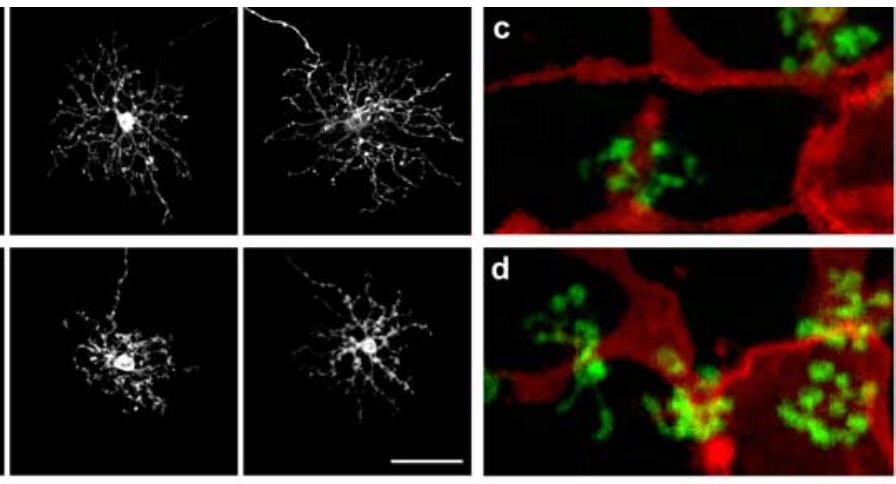

f

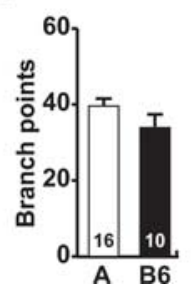

A B6 h

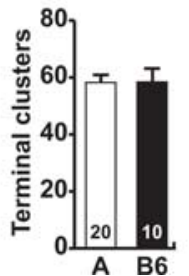

A B6 i

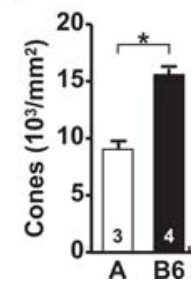

A B6

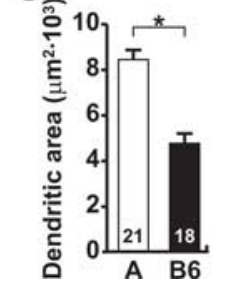

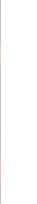

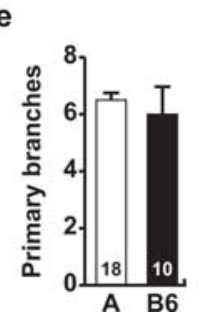

Figure 2. Horizontal cell dendritic fields maintain a constant dendritic coverage across variation in density. $\boldsymbol{a}, \boldsymbol{b}$, Examples of in three adult horizontal cells in two strains of mice, A and B6, respectively. Scale bar: (in $\boldsymbol{b}$ ) $\boldsymbol{a}, \boldsymbol{b}, 50 \mu \mathrm{m}$. $\boldsymbol{c}, \boldsymbol{d}$ (red) and terminath er horizontal cell is identical in the two strains. $\boldsymbol{i}$, Cone density in the A strain is $58 \%$ of that in $\mathrm{B} 6 .{ }^{*} p<0.01 . n=$ the number of mer of retinas analyzed in $\boldsymbol{i}$. Error bars represent $\mathrm{SE}$.

pendent of innervation by cone photoreceptors. Their dendritic patterning, in contrast, is critically dependent on the afferents. At $\mathrm{P} 5$, individual horizontal cells already produce six or seven primary dendrites, some of which extend conspicuously farther than the remainder, from which multiple branches emerge (Fig. 5a). Such $z$-axis reconstructions lose all depth information within the tissue, but Figure 4, $a$ and $f$, shows that their processes have not yet stratified in the presumptive OPL (see also movie 1, available at www.jneurosci.org as supplemental material). These features are essentially unchanged in the coneless retina (Figs. 5b, 6a), although dendritic field extent appears slightly, if not significantly, increased (Fig. 6b). By P10, however, the dendritic fields of horizontal cells deprived of their afferents become conspicuously denser as a result of a more elaborate branching (Figs. $5 c, d, 6 c$ ). Despite this excessive branching in the absence of their afferents, those dendrites become restricted to the OPL, as do horizontal cells in wild-type retinas (Fig. $4 g, h$; see also movie 2 , available at www.jneurosci.org as supplemental material). In addition, although wild-type horizontal cells at P10 show rudimentary terminal clusters that characterize the mature horizontal cell where it contacts a cone pedicle (Fig. $3 b$ ), they are rarely detected in the coneless retina (Figs. $5 c, d, 6 d$ ). Subsequently, the wild-type horizontal cells show relatively little additional morphological differentiation beyond a refinement of their terminal clusters (Figs. $3 a, b, 5 e)$, whereas those in the coneless retina undergo a conspicuous atrophy by adulthood (Fig. $5 f$ ), yielding a sparse dendritic field with meager branching or terminal aggregation (Fig. $6 c, d$; see also movie 3, available at www.jneurosci.org as supplemental material). Note, though, that total dendritic field area is not significantly different in maturity (Fig. 6b). These results show that, rather than being indifferent to the presence of cone afferents, horizontal cells are critically dependent on them for their proper dendritic branching and terminal clustering within the OPL. 
a

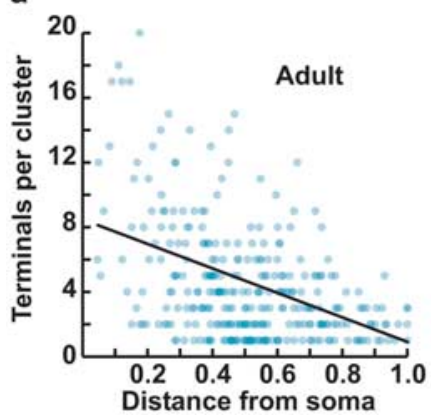

b

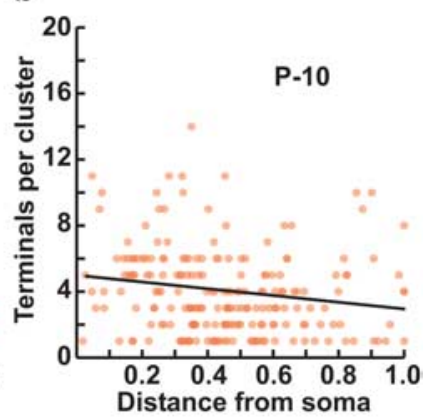

C
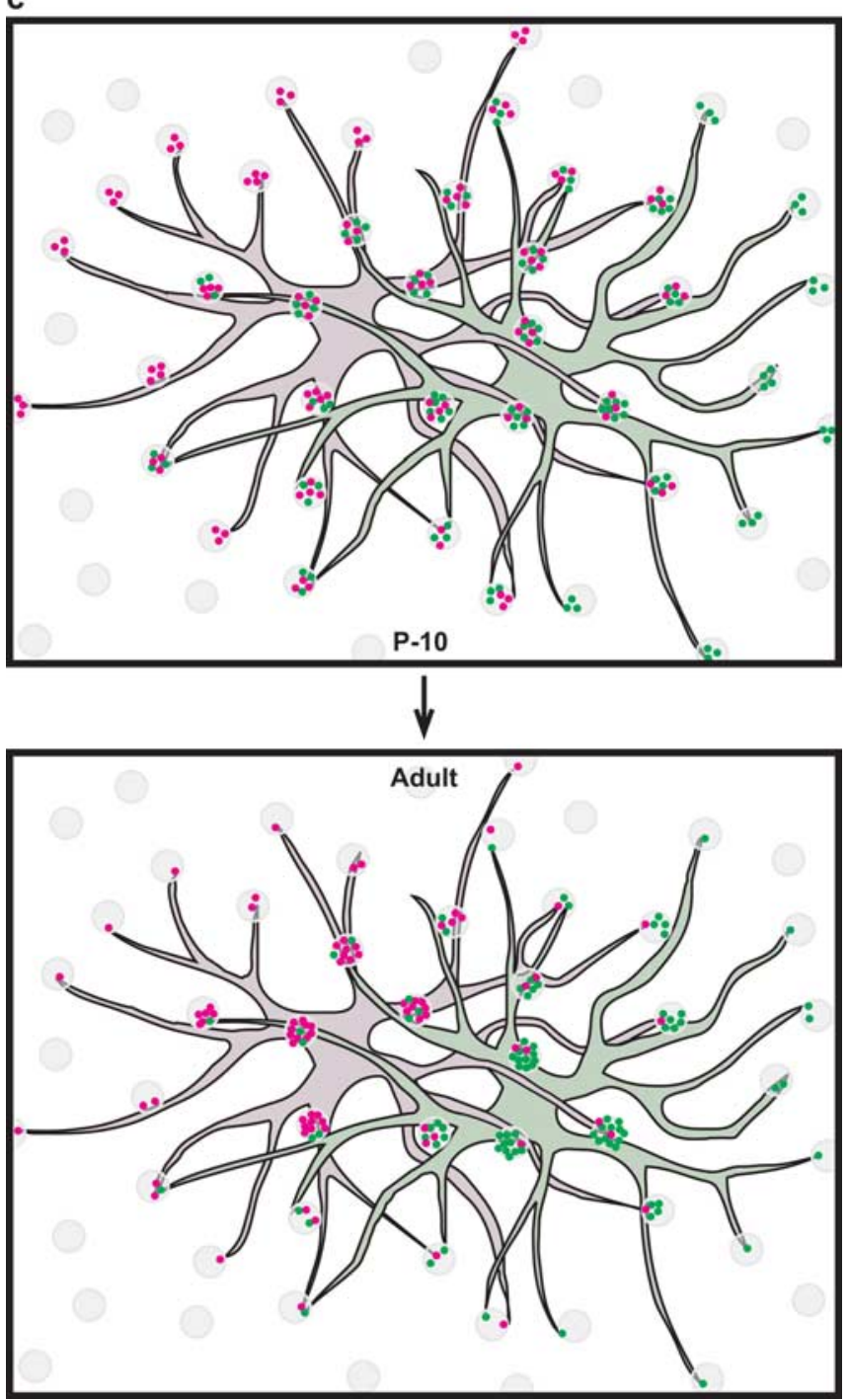

Figure 3. The number of terminals per cluster changes during development as a function of dendritic field extent. $\boldsymbol{a}$, Mature B6 horizontal cells show a steep decline in the number of terminals per pedicle as a function of normalized pedicle distance from the soma. $n=3$ cells. $\boldsymbol{b}$, At P10, in contrast, the relationship is shallow, with relatively fewer terminals at proximal pedicles and more terminals at distal pedicles when compared with the adult. $n=4$ cells. In both $\boldsymbol{a}$ and $\boldsymbol{b}$, the data from individual cells have been pooled. When the slope is calculated for individual cells, and the two groups are then compared, they are significantly different (Student's $t$ test; $p<0.05$ ). c, Because of dendritic overlap, this change in the distribution of terminals for a horizontal cell must be concurrent with that occurring in neighboring horizontal cells (magenta vs green dots), each cell colonizing pedicles (shaded gray) as a function of the distance of its soma to each pedicle relative to other horizontal cells contacting the same pedicle. The schematic shows only two neighboring horizontal cells, but the change depicted at a given pedicle should involve processes from about six horizontal cells.
A few terminal-like aggregates are present in maturity in the coneless retinas, but they appear larger and dystrophic by comparison with those for wild-type horizontal cells (Fig. 7b). They lack discriminable individual terminals that characterize the clustered aggregates in wild-type retina (Fig. 7a). These dystrophic clusters may be postsynaptic to the very few surviving cone pedicles in coneless retinas, but their frequency (Fig. $6 d$ ) is 10 times greater than numerical estimates of the latter (Soucy et al., 1998; Raven and Reese, 2003); rather, their abnormal morphology (Fig. $7 b, d$ ) suggests a de novo dystrophy within the OPL that may be secondary to other plastic changes therein. Various rodent models of inherited retinal degeneration show later-arising alterations in dendritic organization within the OPL (Peng et al., 2000; Strettoi and Pignatelli, 2000; Cuenca et al., 2004), including ectopic synaptic complexes between horizontal cell dendrites with other surviving components of the OPL (Cuenca et al., 2004). The present dystrophies may be an accelerated version of the same, given the relative speed with which the cones are eliminated in these mice.

\section{Discussion}

The present results demonstrate four new findings. First, the positioning of horizontal cells is controlled by mechanisms prohibiting proximity between like-type neighbors, being independent of genotype and scaled to local density. Second, developing horizontal cells extend their dendritic fields to establish comparable dendritic overlap across a twofold variation in horizontal cell density, ensuring a constant coverage of the retina. Third, the connectivity between a horizontal cell and a cone pedicle changes during development, acquiring or eliminating contacts as a function of relative distance. And fourth, cone afferents play a critical, although selective, role in regulating the dendritic morphology of horizontal cells.

\section{Intercellular spacing is independent of genotype}

The mosaic organization of horizontal cells may be attributable to periodic fate-determining events at the time of neurogenesis (Cameron and Carney, 2004), or it may arise secondarily through a process of selective cell death eliminating excessively produced horizontal cells (Cook and Chalupa, 2000; Raven et al., 2003), or it may be a consequence of tangential dispersion as like-type neighbors repel one another (Reese and Galli-Resta, 2002). Any of these developmental mechanisms, or some combination of them, may ultimately account for the anticlustering nature of their distribution. The present results show that, whichever is correct, genetic background does not appear to influence its operation. Rather, the role of genetic background here must be to determine the difference in horizontal cell number, through modulating either horizontal cell production or controlling horizontal cell survival. The present variation in density is clearly not a consequence of differential retinal expansion, because horizontal cell density does not vary appreciably with retinal eccentricity in the mouse (Strettoi and Pignatelli, 2000; Raven and Reese, 2002), and the areal size of the retinas in these different strains do not correlate with average strain density.

The differences in horizontal cell number in the mouse retina are unlikely to be a product of modulating cell death, though, because there is no published evidence that these cells are overproduced during development nor any direct evidence that they die (Young, 1984). Furthermore, for those populations of retinal neuron undergoing naturally occurring cell death, the size of the population can be modulated by manipulating synaptic relationships during development (for review, see Linden, 2000). Elimi- 
nation of the cone afferents, in contrast, does not influence horizontal cell survival (Raven and Reese, 2003). These results would support the alternative, that differential production during the neurogenetic period is the likely cause of the differences between the strains in their horizontal cell density.

There are, however, two reasons to question whether this mechanism, differential production, is sufficient to account for the final patterning in the mosaic of horizontal cells. First, horizontal cell genesis is complete by embryonic day 16 in the mouse, yet by $\mathrm{P} 1$, when the horizontal cells have settled within the neuroblast layer, they disperse tangentially for short distances (Reese et al., 1999). Such dispersion can only change the patterning present at the time of fate assignment, established before migration into the neuroblast layer. That such short-distance movements might improve the patterning of the mosaic is supported by the finding that mosaic regularity improves after horizontal cells settle within the neuroblast layer (Scheibe et al., 1995). Consequently, although fate determination events undoubtedly establish a rudimentary patterning within the population of newly generated horizontal cells, interactions with differentiating neighbors appear to refine their intercellular spacing (Reese and Galli-Resta, 2002).

\section{Dendritic field area is scaled to density}

The present results also suggest that homotypic neighbors interact with one another to constrain the lateral extent of their dendritic fields. Other examples of this form of dendritic control have been suggested (Wässle et al., 1981; Perry and Linden, 1982; Amthor and Oyster, 1995; Grueber et al., 2003), but recent evidence now calls into question this mechanism, at least for two types of retinal ganglion cell (Lin et al., 2004) and for cholinergic amacrine cells (Farajian et al., 2004). How the horizontal cells, with a coverage factor of $\sim 6-7$, coordinate their growth to achieve this dendritic coverage across a wide variation in density between different strains of mice is presently unclear. One simple strategy is for dendritic field growth to be downregulated during contact with neighboring somata, consistent with the fact that the average dendritic field radius for each strain (A, $48.0 \pm 0.23 \mu \mathrm{m}$; $\mathrm{B} 6,35.0 \pm 0.18 \mu \mathrm{m})$ is slightly longer than the average Delauney segment length (that is, the average length between Voronoi neighbors: A, $40.6 \pm 0.59 \mu \mathrm{m}$; B6, $27.8 \pm 0.34 \mu \mathrm{m})$. The extension of, on average, six primary dendrites from the soma would appear well suited to detect approximately five or six Voronoi neighbors surrounding each cell.

\section{Colonization of a pedicle is dependent on the relative proximity of neighboring horizontal cells}

Cone pedicles stimulate horizontal cell dendrites to produce terminals that invaginate into the pedicle beginning at P5 so that by $\mathrm{P} 10$, the number of terminal clusters approximates the number found in maturity. Yet, as development proceeds, the number of terminals in a cluster contributed by a horizontal cell changes after P10, depending on the position of the pedicle relative to the soma. This developmental rearrangement is not simply a loss of terminal contacts at distal sites in the dendritic field; if it were, then this might simply have reflected a cell-intrinsic limitation on maintaining terminals as a function of physical distance. Rather, there is a reciprocal relationship between such a reduction at distal sites with an increase in the number of terminal contacts at a central site in the dendritic field of an adjacent horizontal cell, suggestive of an upper limit on the number of terminals that a pedicle can support (Ahnelt and Kolb, 1994a,b). Horizontal cells may compete in the process of this colonization of the pedicles, with competitive vigor being a function of relative distance to the soma. Such a mechanism, dependent on relative distance, ensures that a constant anatomical coverage at the pedicle will be maintained across variation in density. The change occurs after synaptic triads have formed (Olney, 1968; Blanks et al., 1974) and after photoreceptors become functional (Furukawa et al., 1999; Rohrer et al., 1999), suggesting that visual activity may drive this interaction.

\section{Afferents selectively regulate the dendritic morphology of horizontal cells}

The present results also show that cone afferents regulate selective features of differentiating horizontal cells. Specifically, they control the frequency of higher-order branching and the formation of terminal aggregates at the site of individual cone pedicles. The subsequent reduction in higher-order branching in coneless hor- 


\section{P5}
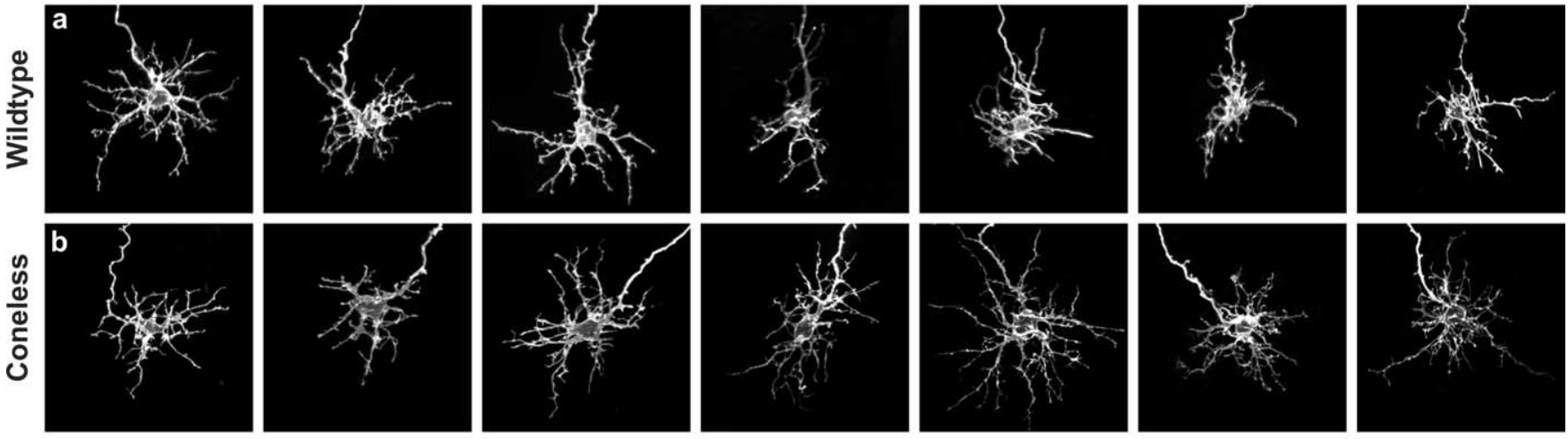

\section{P10}
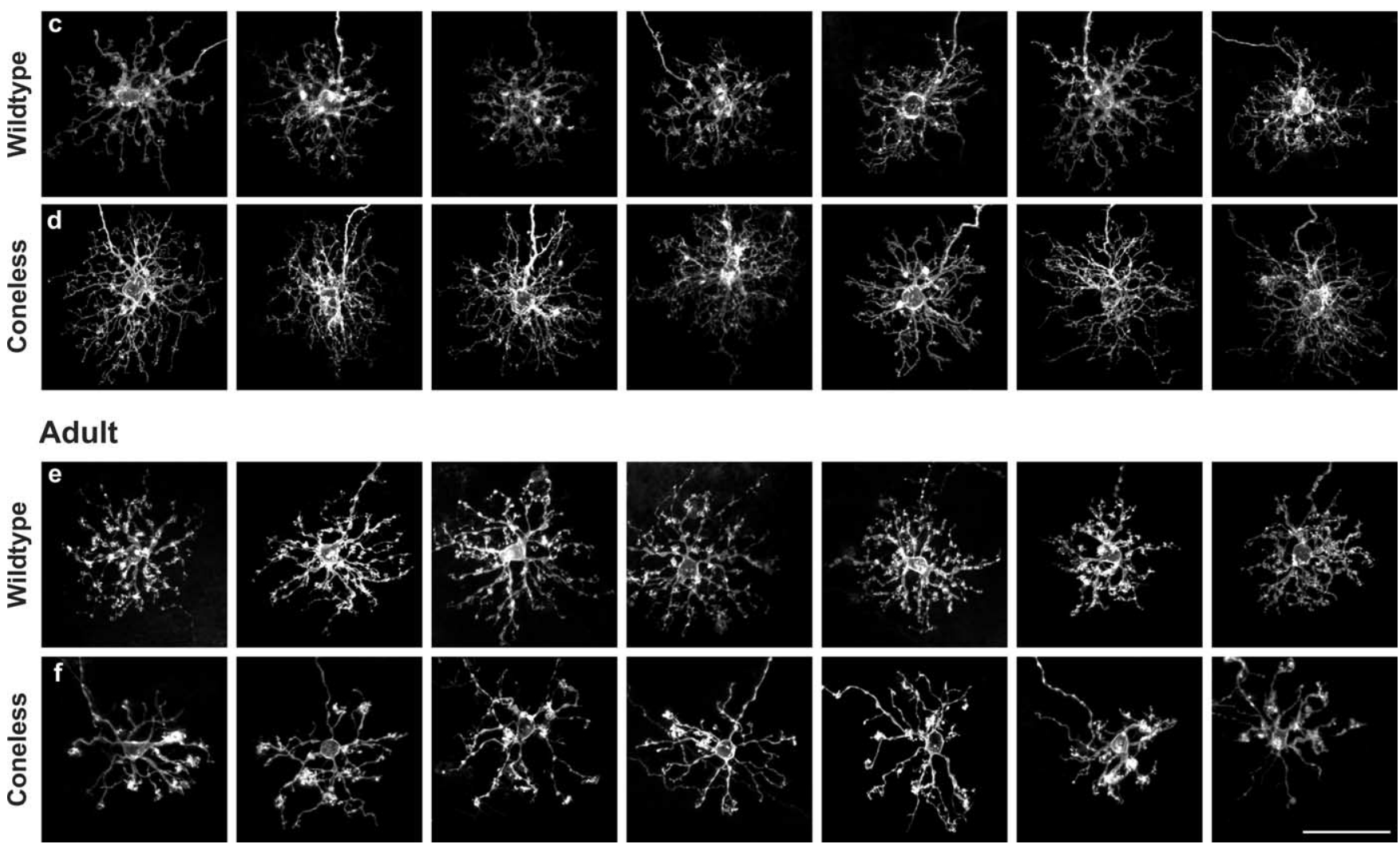

Figure 5. Development of dendritic patterning in wild-type and coneless transgenic mice. $\boldsymbol{a}, \boldsymbol{c}, \boldsymbol{e}$, Morphological development of horizontal cells in wild-type retinas from P5 through adult. $\boldsymbol{b}, \boldsymbol{d}$, $\boldsymbol{f}$, Altered dendritic development in coneless transgenic retinas. Scale bar: (in $\boldsymbol{f}$ ) $\boldsymbol{a}-\boldsymbol{f}, 50 \mu \mathrm{m}$.

izontal cells suggests a critical period for afferent innervation, beyond which such regressive events are triggered. The dendritic terminal aggregates, in comparison, do not form in the absence of the afferents and presumably also require the afferents for their maintenance (Cuenca et al., 2004). Although these features of dendritic patterning are clearly dependent on the afferents, overall dendritic field area, like the number of primary dendrites, is independent of afferent input.

Horizontal cell dendrites normally redistribute to the developing OPL after cone afferents have become stratified. Unexpectedly, this dendritic stratification continued to emerge in the absence of a stratified population of cone pedicles. Although the horizontal cells may have some intrinsic instruction for adopting such a morphology, environmental signals must define the positioning of those dendrites with respect to retinal depth. The OPL is not present when horizontal cells begin this transformation, forming at about the time that the horizontal processes coalesce within a distinct stratum. Because synaptogenesis between the pedicles and horizontal cells may be initiated slightly before this (Blanks et al., 1974; Rich et al., 1997), it remains possible that contact with the cones, even before their pedicles become stratified, triggers this morphological change, but their subsequent stratification at this depth would still require explanation. Some other signal may already presage the positioning of the OPL, because the horizontal cells settle at this depth within the neuroblast layer as early as the day of birth.

\section{Conclusions}

The present results show how different features of the dendritic morphology of horizontal cells are controlled independently dur- 
a
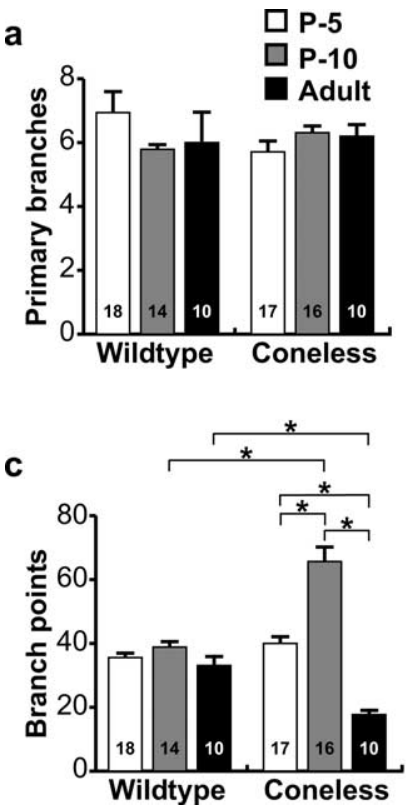

b

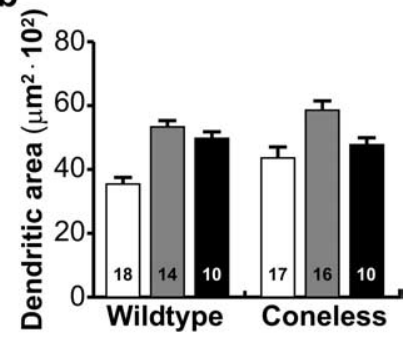

d

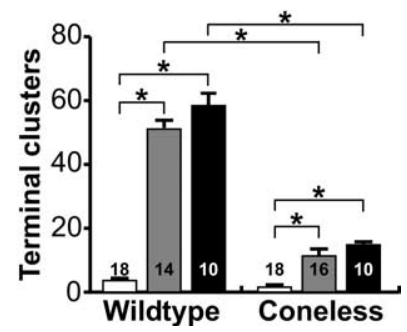

Figure 6. Afferent elimination perturbs dendritic branching and terminal differentiation of horizontal cells. $\boldsymbol{a}$, The number of primary dendrites was not significantly different between horizontal cells from wild-type and coneless retinas. $\boldsymbol{b}$, Dendritic field area likewise was not significantly between the two conditions. c, Frequency of branching, however, was significantly greater at P10 in the coneless retina, whereas it subsequently became significantly reduced in the adult. $\boldsymbol{d}$, The formation of terminal clusters normally occurs between P5 and P10; coneless transgenic retinas fail to form appreciable numbers of these aggregated terminals at any age. ${ }^{*} p<0.01 . n=$ the number of cells analyzed in each condition. Error bars represent SE.

\section{Wildtype}
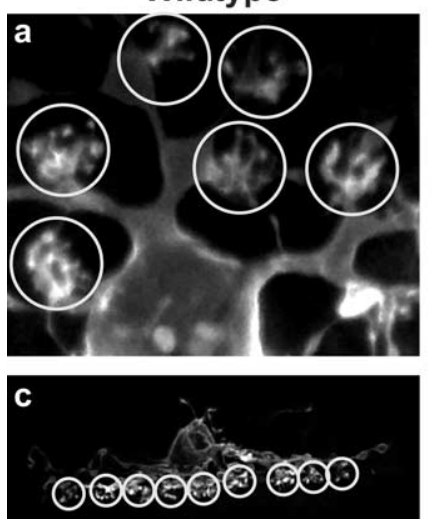

\section{Coneless}
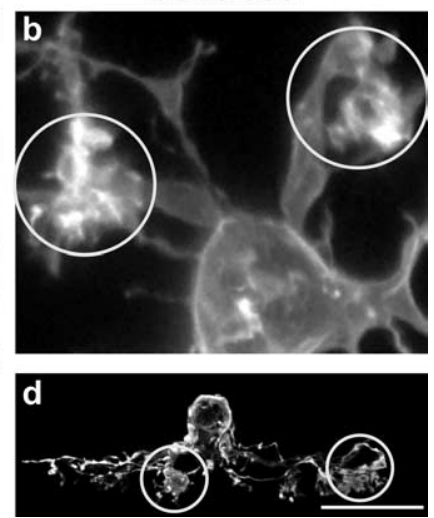

Figure 7. The few terminal clusters (circles) that form in coneless transgenic retinas are large and dystrophic $(\boldsymbol{b}, \boldsymbol{d})$ when compared with those in wild-type retinas $(\boldsymbol{a}, \boldsymbol{c})$. Images in $\boldsymbol{c}$ and $\boldsymbol{d}$ are reconstructed from 27 adjacent $1 \mu \mathrm{m}$ confocal planes through the center of the field. Scale bar: (in d) $\boldsymbol{a}, \boldsymbol{b}, 9 \mu \mathrm{m} ; \boldsymbol{c}, \boldsymbol{d}, 25 \mu \mathrm{m}$.

ing development. Primary dendritic branches form before afferent innervation and are maintained independent of those afferents, likely reflecting cell-intrinsic specification. The length of those primary dendrites, in contrast, is determined by proximity to neighboring horizontal cells. Higher-order branching is subsequently regulated by the afferents, but the stratification of those branches does not require innervation from the afferents. Afferents also induce the formation of terminal clusters, but the final colonization of a pedicle by dendritic terminals from different horizontal cells is dependent on their relative proximity to the pedicle. Intercellular spacing is regulated by homotypic interactions occurring at the earliest stages, when horizontal cells first

settle within the neuroblast layer and disperse to space themselves out well before the afferents stratify. Multiple environmental signals therefore modulate an intrinsic program to shape the morphology, positioning, connectivity, and coverage of the horizontal cell network.

\section{References}

Ahnelt P, Kolb H (1994a) Horizontal cells and cone photoreceptors in primate retina: a Golgi-light microscopic study of spectral connectivity. J Comp Neurol 343:387-405.

Ahnelt P, Kolb H (1994b) Horizontal cells and cone photoreceptors in human retina: a Golgi-electron microscopic study of spectral connectivity. J Comp Neurol 343:406-427.

Amthor FR, Oyster CW (1995) Spatial organization of retinal information about the direction of image motion. Proc Natl Acad Sci 92:4002-4005.

Blanks JC, Adinolfi AM, Lolley RN (1974) Synaptogenesis in the photoreceptor terminal of the mouse retina. J Comp Neurol 156:81-93.

Cameron DA, Carney LH (2004) Cellular patterns in the inner retina of adult zebrafish: quantitative analyses and a computational model of their formation. J Comp Neurol 471:11-25.

Cellerino A, Novelli E, Galli-Resta L (2000) Retinal ganglion cells with $\mathrm{NADPH}$-diaphorase activity in the chick form a regular mosaic with a strong dorsoventral asymmetry that can be modelled by a minimal spacing rule. Eur J Neurosci 12:613-620.

Cline HT (2001) Dendritic arbor development and synaptogenesis. Curr Opin Neurobiol 11:118-126.

Cook JE, Chalupa LM (2000) Retinal mosaics: new insights into an old concept. Trends Neurosci 23:26-34.

Cuenca N, Pinilla I, Sauvé Y, Lu B, Wang S, Lund RD (2004) Regressive and reactive changes in the connectivity patterns of rod and cone pathways of P23H transgenic rat retina. Neuroscience 127:301-317.

Farajian R, Raven MA, Cusato K, Reese BE (2004) Cellular positioning and dendritic field size of cholinergic amacrine cells are impervious to early ablation of neighboring cells in the mouse retina. Vis Neurosci 21:13-22.

Fei Y (2003) Development of the cone photoreceptor mosaic in the mouse retina revealed by fluorescent cones in transgenic mice. Mol Vis 9:31-42.

Furukawa T, Morrow EM, Li T, Davis FC, Cepko CL (1999) Retinopathy and attenuated circadian entrainment in $\mathrm{Cr} x$-deficient mice. Nat Genet 23:466-470.

Grueber WB, Ye B, Moore AW, Jan LY, Jan YN (2003) Dendrites of distinct classes of Drosophila sensory neurons show different capacities for homotypic repulsion. Curr Biol 13:618-626.

Hua JY, Smith SJ (2004) Neural activity and the dynamics of central nervous system development. Nat Neurosci 7:327-332.

Jan Y-N, Jan LY (2003) The control of dendrite development. Neuron 40:229-242.

Komiyama T, Johnson WA, Luo L, Jefferis GS (2003) From lineage to wiring specificity. POU domain transcription factors control precise connections of Drosophila olfactory projection neurons. Cell 112:157-167.

Lia B, Williams RW, Chalupa LM (1987) Formation of retinal ganglion cell topography during prenatal development. Science 236:848-851.

Lin B, Wang SW, Masland RH (2004) Retinal ganglion cell type, size, and spacing can be specified independent of homotypic dendritic contacts. Neuron 43:475-485.

Linden R (2000) The anti-death league: associative control of apoptosis in developing retinal tissue. Brain Res Rev 32:146-158.

Lom B, Cogen J, Sanchez AL, Vu T, Cohen-Cory S (2002) Local and targetderived brain-derived neurotrophic factor exert opposing effects on the dendritic arborization of retinal ganglion cells in vivo. J Neurosci 22:7639-7649.

Olney JW (1968) An electron microscopic study of synapse formation, receptor outer segment development, and other aspects of developing mouse retina. Invest Ophthalmol 7:250-268.

Peichl L, González-Soriano J (1994) Morphological types of horizontal cell in rodent retinae: a comparison of rat, mouse, gerbil, and guinea pig. Vis Neurosci 11:501-517.

Peichl L, Sandmann D, Boycott BB (1998) Comparative anatomy and function of mammalian horizontal cells. In: Development and organization of the retina (Chalupa LM, Finlay BL, eds). New York: Plenum.

Peng Y-W, Hao Y, Petters RM, Wong F (2000) Ectopic synaptogenesis in the mammalian retina caused by rod photoreceptor-specific mutations. Nat Neurosci 3:1121-1127. 
Perry VH, Linden R (1982) Evidence for dendritic competition in the developing retina. Nature 297:683-685.

Raven MA, Reese BE (2002) Horizontal cell density and mosaic regularity in pigmented and albino mouse retina. J Comp Neurol 454:168-176.

Raven MA, Reese BE (2003) Mosaic regularity of horizontal cells in the mouse retina is independent of cone photoreceptor innervation. Invest Ophthalmol Vis Sci 44:965-973.

Raven MA, Eglen SJ, Ohab JJ, Reese BE (2003) Determinants of the exclusion zone in dopaminergic amacrine cell mosaics. J Comp Neurol 461:123-136.

Reese BE, Galli-Resta L (2002) The role of tangential dispersion in retinal mosaic formation. Prog Ret Eye Res 21:153-168.

Reese BE, Necessary BD, Tam PPL, Faulkner-Jones B, Tan S-S (1999) Clonal expansion and cell dispersion in the developing mouse retina. Eur J Neurosci 11:2965-2978.

Rich KA, Zhan Y, Blanks JC (1997) Migration and synaptogenesis of cone photoreceptors in the developing mouse retina. J Comp Neurol 388:47-63.

Rodieck RW (1991) The density recovery profile: a method for the analysis of points in the plane applicable to retinal studies. Vis Neurosci 6:95-111.

Rodieck RW, Marshak DW (1992) Spatial density and distribution of choline acetyltransferase immunoreactive cells in human, macaque, and baboon retinas. J Comp Neurol 321:46-64.

Rohrer B, Korenbrot JI, LaVail MM, Reichardt LF, Xu B (1999) Role of neurotrophin receptor TrkB in the maturation of rod photoreceptors and establishment of synaptic transmission to the inner retina. J Neurosci 19:8919-8930.

Sandmann D, Boycott BB, Peichl L (1996) Blue-cone horizontal cells in the retinae of horses and other Equidae. J Neurosci 16:3381-3396.
Scheibe R, Schnitzer J, Rohrenbeck J, Wohlrab F, Reichenbach A (1995) Development of A-type (axonless) horizontal cells in the rabbit retina. J Comp Neurol 354:438-458.

Scott EK, Luo L (2001) How do dendrites take their shape? Nat Neurosci 4:359-365.

Soucy E, Wang Y, Nirenberg S, Nathans J, Meister M (1998) A novel signaling pathway from rod photoreceptors to ganglion cells in mammalian retina. Neuron 21:481-493.

Strettoi E, Pignatelli V (2000) Modifications of retinal neurons in a mouse model of retinitis pigmentosa. Proc Natl Acad Sci USA 97: 11021-11025.

Wang Y, Macke JP, Merbs SL, Zack DJ, Klaunberg B, Bennett J, Gearhart J, Nathans J (1992) A locus control region adjacent to the human red and green visual pigment genes. Neuron 9:429-440.

Wässle H, Peichl L, Boycott BB (1981) Morphology and topography of onand off-alpha cells in the cat retina. Proc R Soc Lond B Biol Sci 212:157-175.

Wässle H, Röhrenbeck J, Boycott BB (1989) Horizontal cells in the monkey retina: cone connections and dendritic network. Eur J Neurosci 1:421-435.

Williams RW, Strom RC, Zhou G, Yan Z (1998) Genetic dissection of retinal development. Semin Cell Dev Biol 9:249-255.

Wong ROL, Ghosh A (2002) Activity-dependent regulation of dendritic growth and patterning. Nat Rev Neurosci 3:803-812.

Young RW (1984) Cell death during differentiation of the retina in the mouse. J Comp Neurol 229:362-373.

$\mathrm{Yu}$ X, Malenka RC (2003) $\beta$-Catenin is critical for dendritic morphogenesis. Nat Neurosci 6:1169-1177. 